\title{
BRINCANDO COM EUCLIDES: O INFINITAMENTE DIVISÍVEL NAS PROPOSIÇÕES 7 E 8 DO ELEMENTO 2
}

\author{
Aron Seixas-Aluno-ICT/UFVJM-aronseixas@yahoo.com.br \\ Cíntia M. Hamada-Aluna-ICT/UFVJM-cintia-hamada@ hotmail.com \\ Débora Pelli-T.A.-ICT/UFVJM-debora.pelli@ufvjm.edu.br \\ Douglas F. Santiago-Professor Ms.-ICT/UFVJM-douglas.santiago@ufvjm.edu.br \\ Jéssica E. Oliveira-Aluna-ICT/UFVJM-jessicaeoliveira@ hotmail.com \\ José Lucas de Camargos-Aluno-Eng. Florestal/UFVJM-jlcamargos@yahoo.com.br \\ Leidiane Aparecida da Silva-Aluna-ICT/UFVJM-leidileidiane@yahoo.com.br \\ Lilian V. Santos-Aluna bolsista REUNI-ICT/UFVJM-vieirasantoslilian@yahoo.com.br \\ Orlindo W. Pereira-Aluno-ICT/UFVJM-orlindowagner@ gmail.com \\ Raquel A. Sapunaru-Professora Dra.-raquel.sapunaru@ufvjm.edu.br (coordenadora-contato) \\ Stéphany S. Souza-Aluna-ICT/UFVJM-sspss_05@ hotmail.com
}

\begin{abstract}
Resumo: O presente artigo almeja discutir a possibilidade de existir em algumas proposições da obra Os Elementos de Euclides indícios da divisão infinita utilizada por Leibniz no estabelecimento dos Cálculos Diferencial e Integral. Para tal, o grupo trabalha inicialmente com uma análise da principal ideia contida nos elementos, a saber: a discussão do mundo físico-matemático a partir de diagramas. Estas imagens estabeleceram um método único e incomparável que serviram de base para Leibniz (entre outros pensadores) no desenvolvimento de seu próprio pensamento. Particularmente, nós nos concentramos nas análises das proposições 7 e 8 do livro 2 e, das proposições 17 e 18 do livro 3. Além disso, nós utilizamos o texto de Leibniz onde ele estabeleceu as regras de derivação e integração e apresentou seu método de máximos e mínimos. Grosso modo, a utilização das proposições de Euclides mencionadas em combinação com os escritos de Leibniz pretende proporcionar uma maior compreensão do infinitamente pequeno.
\end{abstract}

Palavras-chave: Euclides. Leibniz. Geometria. Imagem. Infinitesimal.

\begin{abstract}
This paper aims to discuss the possibility of work in some in propositions of Euclid's Elements in infinite division signs used by Leibniz in the establishment of differential and integral calculus. To this end, "The" group works with at first with an analysis of the main idea contained in the elements, namely the discussion of the mathematicalphysical world from images. These images have established a unique and unparalleled method that served as the basis for Leibniz (among other thinkers) to develop their own thought. Especially, we focus on the analysis of propositions 7 and 8 of Book 2, and of propositions 17 and 18 of book 3. In addition, we use Leibniz's text where he established the rules of derivation and integration, and presented his method of maxima and minima. Roughly speaking, the use of Euclid's propositions mentioned in conjunction with the writings of Leibniz intends to provide a greater understanding of the infinitely small.
\end{abstract}

Keywords: Euclides. Leibniz. Geometria. Imagem. Infinitesimal. 


\section{Introdução}

A geometria euclidiana pode ser despretensiosamente definida como aquela com a qual nós nos acostumamos a sentir e entender o mundo, desde sempre. O espaço, contudo, segundo concepções modernas não é plano, é curvo e, as coisas não são o que parecem ser, porque elas estão além de nossos sentidos. A obviedade euclidiana não existe mais. No entanto, a geometria de Euclides foi construída em bases sólidas, não somente sob o dogma dos sentidos, mas também sob a lupa da razão que adentra as sensações. Não é à toa que as ideias de Euclides inspiraram e inspirarão inúmeros pensadores da história da humanidade.

Curiosamente, Euclides não usa números: sua linguagem é imagética, isto é, passa da imaginação para a imagem representativa da realidade sensível e viceversa, sem medo de se perder num labirinto de curvas, ângulos e retas. Para Euclides (e em Euclides) não há como se perder, pois é na imagem do real, no diagrama ou esquema, traduzido em definições, axiomas ou postulados e teoremas que encontramos a beleza que enfeitiçou e introduziu uma prática comum a diversos pensadores, entre os quais destacamos Leibniz. Através deste filósofo, a prática da matematização revelou-se no pensar, entender e explicar o mundo geometricamente. Do ponto de vista de
Leibniz, para entender um pouco mais sobre essa imagem representativa do mundo e de tudo mais que nele estava contido, incluindo o próprio pensamento que a criou para representá-lo, era preciso seguir também um método igualmente poderoso e soberbo. Euclides, como o inventor do método axiomático, tornou-se um vulto de referência, pois o livro Os Elementos é considerado o primeiro sistema lógico seguido por ciências e cientistas, até os dias de hoje. (EVES, 2004, p.178-180) Todavia, não podemos ignorar que Euclides se esforçou muito para axiomatizar a geometria com os meios de que dispunha em sua época. Porém, observamos que em algumas demonstrações Euclides admitiu resultados puramente intuitivos, sem fazer uso de demonstrações. Neste ponto é que a importância do desenho entra em cena.

No método euclidiano, a prática da construção de diagramas para explicar as coisas do mundo nos remete a uma universalidade e, esta universalidade provoca necessariamente a construção de imagens reveladas. A epístola passada por Euclides é clara, aberta, quase profética e nela nada há de subliminar: ela atua como um instrumento ordinário, usado para a realização de um trabalho, capaz de nos conduzir a fatos distintos e incontestáveis. Prontamente, as imagens do uso de Euclides agem e interagem como suporte (muletas cerebrais), pois através delas é possível aumentar o escopo de 
entendimento das argumentações que nos remetem as verdades necessárias. Igualmente, é na geometria das imagens euclidianas que as disputas metodológicas desaparecem, perdem o sentido, pois nela localizamos pela primeira vez, de modo complementar, os métodos de análise e síntese. No universo euclidiano, transportado através dos séculos, o método analítico reduziu complexas construções, como emaranhados de linhas pontos, retas e cortes, a seus simples constituintes e, neste mesmo caminho, a síntese encarregou-se de reconstruir estas mesmas complexas construções a partir destes constituintes simples, recém apresentados. Por fim, Euclides e todos aqueles que utilizaram seus conhecimentos entenderam que a análise era um refluxo ao simples; e, portanto, a síntese não poderia ser outra coisa diferente da progressão do simples em direção ao complexo.

Destarte, diante do poder da linguagem imagética que pode ser a causa ou o efeito da conjunção dos métodos analítico e sintético, pensamos ser impossível arquitetar um mundo sem Euclides, pois sem ele teria sido impossível para Leibniz, chegar aos resultados que chegou. No entanto, uma dúvida permanece: mesmo com o incontestável desenvolvimento do cálculo a partir dos escritos de Cavalieri (EVES, 2004, p.425-428) é estimulante especular como Leibniz teria conseguido consolidar a ideia de um infinitamente pequeno. Teria ele se inspirado em Euclides? Colocando de outro modo, poderíamos pensar que o infinitamente pequeno pudesse aparecer, recondidamente, em algumas poucas proposições de alguns dos treze elementos? É certo que Euclides não possuía e nem poderia possuir uma noção de infinito como a que herdamos de Leibniz. Porém, os questionamentos que surgiram ao longo dos estudos do grupo "Euclides para os Vales" são legítimos e amparados filosoficamente por Heidegger em seu livro $O$ Princípio da Razão:

\footnotetext{
Quanto maior a obra do pensador - o que não coincide de forma alguma com a amplitude e o número de escritos - mais rico é aquilo que não foi pensado nela, ou seja, aquilo que emerge de dentro e através dela como não tendo ainda sido pensado. É claro que esse não pensado não tem nada a ver com aquilo que o pensador não viu ou não dominou e que descendentes mais sábios teriam então que demonstrar. (HEIDEGGER, 1996, p.71)
}

Norteados por este pensamento, o objetivo deste artigo é mostrar que nas proposições 7 e 8 do elemento 2 de Euclides, encontra-se, mesmo que de modo idealizado e embrionário, as primeiras notas do infinitamente pequeno de Leibniz.

\section{O caminho do infinitamente pequeno: as proposições 7 e 8 , livro 2 , dos} Elementos de Euclides 
Retomando, a importância ímpar do livro Os Elementos de Euclides, é mister nos aprofundarmos um pouco mais na questão de método euclidiano. Conforme a filósofa Olga Pombo, a geometria euclidiana é um sistema lógico.

(http://www.educ.fc.ul.pt/docentes/opombo/se minario/euclides/index.htm) Ademais, entendemos que o método euclidiano, como um sistema lógico, tem por obrigação respeitar regras e normas que, se contrariadas, comprometem o entendimento de tudo que dele infere. Assim, analisando como verdadeira essa afirmação, Olga Pombo corrobora nossa posição quanto à importância do método euclidiano para o desenvolvimento de outras ferramentas matemáticas. Nas palavras de Olga Pombo:

As definições, os axiomas ou postulados (conceitos e proposições admitidos sem demonstração que constituem os fundamentos especificamente geométricos e fixam a existência dos entes fundamentais: ponto, recta e plano) e os teoremas não aparecem agrupados ao acaso, mas antes expostos numa ordem perfeita. Cada teorema resulta das definições, dos axiomas e dos teoremas anteriores, de acordo com uma demonstração rigorosa.

(http://www.educ.fc.ul.pt/docentes/opombo/se $\underline{\text { minario/euclides/index.htm) }}$

Assim, seguindo os passos do livro 1, no livro 2, proposições 7 e 8 , Euclides introduziu uma série de postulados, usou alguns para obter os resultados, intuiu muitas coisas através de suas figuras e, como intencionamos mostrar, poderia ter ido muito mais além. ${ }^{1}$ A seguir, descreveremos as proposições 7 e 8 do livro 2, seguidas de uma análise que aponta para o objetivo deste artigo, ou seja, para a possibilidade de abstrair o infinitamente pequeno da ideia de Euclides, através de divisões infinitas.

\subsection{Proposição 7}

Se um segmento $A B$ é dividido em dois por um ponto $\mathrm{C}$, então o quadrado sobre o lado AB mais o quadrado sobre o lado CB é igual a duas vezes o retângulo de lados $A B$ e CB mais o quadrado sobre o lado AC. (EUCLIDES, 2009, p.141)

A partir do segmento $A B$ vamos fazer a seguinte construção:

1. Tome um ponto $\mathrm{C}$ dividindo $\mathrm{o}$ segmento em duas partes. Desta forma, temos os pontos $\mathrm{A}, \mathrm{C}$ e $\mathrm{B}$, nesta ordem, sobre a mesma reta.

2. Tendo como lado o segmento $A B$, construa o quadrado ADEB (pontos no sentido anti-horário) e trace a diagonal $\mathrm{BD}$.

3. Pelo ponto $\mathrm{C}$, construiremos uma paralela ao lado BE do quadrado. Esta paralela encontrara a diagonal $\mathrm{BD}$ em um ponto $\mathrm{F}$ e o lado oposto do quadrado em um ponto G. A partir deste ponto $\mathrm{F}$ de intercessão, construa uma paralela ao lado $\mathrm{AB}$. 
Esta encontra o lado AD do quadrado no ponto $\mathrm{H}$ e o lado oposto, BE, no ponto I.

Seguindo os passos descritos no Diagrama 1, nosso quadrado ADEB é o quadrado de lado $\mathrm{AB}$ dividido em quatro partes. Ao longo da diagonal, os quadrados BF e FD (realmente quadrados conforme proposição anterior) e as figuras retangulares $\mathrm{AF}$ e $\mathrm{FE}$, têm a mesma área. $\mathrm{O}$ fato das áreas de AF e FE serem iguais faz com que as áreas dos retângulos AI e CE também sejam iguais, pois os últimos são formados acrescentando a $\mathrm{AF}$ e $\mathrm{FE}$ o mesmo quadrado $\mathrm{CI}$ que possui área igual ao quadrado de lado CB. Todavia, o retângulo AI tem área igual a do retângulo formado pelos lados AB e CB. Assim, se somarmos duas vezes a área do retângulo de lados $\mathrm{AB}$ e $\mathrm{CB}$ (correspondente a $\mathrm{AI}+\mathrm{CE}$ ) a área do quadrado de lado AC (correspondente a FD), obteremos a área do quadrado de lado $\mathrm{AB}$ mais a área do quadrado de lado $\mathrm{CB}$ (correspondente ao CI). QED.

Diagrama 1- Proposição 7 do livro 2 dos Elementos de Euclides

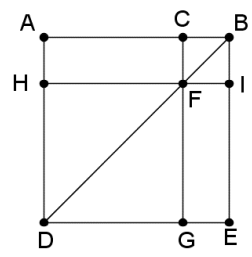

Adaptação livre (EUCLIDES, 2009, p.141)

Para nós, ficou claro que quaisquer partições extras feitas nesses retângulos e quadrado não trazem nenhuma alteração no somatório de suas áreas.

\subsection{Proposição 8}

Se um segmento $\mathrm{AB}$ é dividido em duas partes por um ponto $\mathrm{C}$, então quatro vezes o retângulo de lados $\mathrm{AB}$ e $\mathrm{CB}$ mais o quadrado de lado AC é igual ao quadrado de lado AB + CB. (EUCLIDES, 2009, p.142143)

A partir do segmento $A B$ faça a seguinte construção:

1. Tome um ponto $\mathrm{C}$ entre $\mathrm{A}$ e $\mathrm{B}$, ficando assim, na ordem, os pontos A, C e B sobre a mesma reta e, depois estenda esta reta até um ponto D, ficando então, nesta ordem, os pontos $\mathrm{A}, \mathrm{C}, \mathrm{B}$ e $\mathrm{D}$, sendo $\mathrm{CB}=\mathrm{BD}$. Repare que fazendo desta forma, a área do quadrado de lado AD corresponde exatamente à área do quadrado de lado $\mathrm{AB}+\mathrm{CB}$, tendo como vértices $\mathrm{ADFE}$ (sentido horário).

2. Construa um quadrado tendo por lado o segmento $\mathrm{AD}$ e trace a diagonal DE. Pelos pontos C e B, faça paralelas ao lado DF, sendo que a paralela no ponto $\mathrm{C}$ encontra a diagonal traçada no ponto $\mathrm{G}$ e o lado oposto do quadrado no ponto H. A paralela pelo ponto B encontra a diagonal traçada no ponto I e o lado oposto do 
quadrado no ponto J. Tendo por base os pontos de intercessão, I e $\mathrm{G}$, construa outras duas paralelas, agora ao lado AD. A paralela pelo ponto I vai encontrar o lado AE, a paralela $\mathrm{CH}$ e o lado $\mathrm{DF}$ respectivamente nos pontos $\mathrm{K}, \mathrm{L}$ e M. A paralela pelo ponto $G$ vai encontrar o lado AE, a paralela BJ e o lado DF respectivamente, nos pontos $\mathrm{N}, \mathrm{O}$ e $\mathrm{P}$ (repare que é a mesma construção do item 3 da proposição 7, só que agora com dois pontos).

Fazendo da forma descrita, nosso quadrado de lado AD fica dividido em nove partes retangulares. Ao longo da diagonal, temos os quadrados EG, GI e ID. O quadrado EG tem área igual a do quadrado de lado AC. A partir dos quadrados GI e ID, usando resultados já demonstrados e o fato de que $\mathrm{CB}$ $=\mathrm{BD}$, consegue-se mostrar que os quatro quadrados, GI, ID, CI e IP são iguais. Além destes cinco quadrados já descritos, o quadrado de lado AD também contém quatro retângulos, AL, KG, GJ e OF. Juntando AL com CI, obtemos o retângulo AI cuja área é igual a do retângulo IF (mostrado anteriormente), formado pela união de $\mathrm{OF}$ com IP. Porém, a área de IP é a mesma de CI. Logo, a área de OF é igual à de $\mathrm{AL}$ e, por serem figuras retangulares de mesmo lado, também têm a mesma área de KG e GJ. Chegamos então à seguinte conclusão: formando as figuras das seguintes uniões, $\mathrm{AL}$ com CI, IP com OF, GI com GJ e KG com ID, temos quatro retângulos, cada um com área igual ao com lados $\mathrm{AB}$ e $\mathrm{BC}$. Estes quatro retângulos somados com o quadrado $\mathrm{EG}$, de área igual ao de lado $\mathrm{AC}$, formam o quadrado de lado $\mathrm{AD}$, de área igual ao quadrado de lado $\mathrm{AB}+\mathrm{CB}$. $Q E D$.

Diagrama 2- Proposição 8 do livro 2 dos Elementos de Euclides

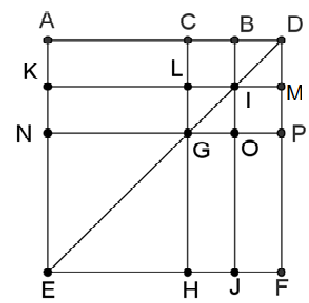

Adaptação livre (EUCLIDES, 2009, p.142)

Partindo da mesma figura da proposição 7, foram escolhidos os pontos médios dos lados AN, DF, CD e HJ dos retângulos (os mesmos da proposição 7), embora estejam com nomes diferentes. Por exemplo, o ponto B da proposição 7 tomou o nome de ponto $\mathrm{D}$ na proposição 8 , assim como o ponto $\mathrm{E}$ na proposição 7 tomou o nome de ponto $F$ na proposição 8 . Por esses pontos médios foram traçados os segmentos KH e BJ, o que não alterou a figura em sua forma inicial. Isto nos leva a concluir também que o somatório das áreas dos quatro retângulos ACLK, KLGN, CBJH, BDFJ somadas ao somatório das áreas dos quatros quadrados CBIL, BDHI, LIOG e IHPO, continua a mesma da área do quadrado grande 
NGHE. Assim, nota-se que infinitamente, ou seja, numa operação infinita, podemos pegar os pontos médios dos novos retângulos e ir fazendo partições, pois o somatório das áreas será sempre igual à área do quadrado maior NGHE. Assim, acreditamos que a análise destas duas proposições em sequência seja o suficiente para afirmarmos que Euclides estava no caminho da divisão infinita. Talvez Cavalieri tenha chegado à mesma conclusão que nós e, simplesmente continuou o trabalho de Euclides, abrindo as portas para o fecho de Leibniz e Newton. (EVES, 2004, p.442-444)

\section{A iluminação de Leibniz: o cálculo infinitesimal fundado nas proposições $17 \mathrm{e}$}

\section{8, livro 3, dos Elementos de Euclides}

Leibniz deu o passo decisivo que levou a invenção do cálculo infinitesimal em outubro de 1675, num texto sem título, através do uso exaustivo da geometria euclidiana. Ele percebeu que os problemas que envolviam o inverso da tangente poderiam se reduzir aos de quadraturas, pois estes problemas pediam a solução da equação de uma curva quando se conhecesse a lei que definia sua tangente. ${ }^{2}$ Assim, analisando o conceito de diferencial, dv, criado por Leibniz, chegamos à seguinte interpretação:

Seja AX uma reta (eixo x), C uma curva e V um ponto nesta curva, conforme o Diagrama 3. Trace dois segmentos de retas, ambas passando por $\mathrm{V}$, um perpendicular a $\mathrm{AX}$, cortando AX em $\mathrm{B}$ e outro tangente a curva C, cortando AX em D. Desta forma, dado um segmento $\mathrm{dx}$ qualquer, $\mathrm{dv}$ será o segmento satisfazendo a relação: $d v / d x=$ $\mathrm{VB} / \mathrm{BD}$

A partir desta interpretação podemos fazer dois comentários interessantes:

1. Consideramos $\theta$ o ângulo que a tangente faz com $\mathrm{AX}$, temos que o valor $\mathrm{dv} / \mathrm{dx}$ é justamente $\operatorname{tg}(\theta)$, isto é, o coeficiente angular da reta tangente, que corresponde ao conceito de derivada.

2. Consideramos que $\mathrm{dx}$ é um segmento que pode variar seu comprimento, conseguimos enxergar $d v$ como o resultado de uma transformação linear que leva $\mathrm{dx}$ em (VB/BD)dx e, para dx cada vez menor, espera-se que $d v$ corresponda a uma estimativa cada vez mais apurada do quanto à curva $\mathrm{C}$ varia na direção perpendicular ao eixo $\mathrm{AX}$, isto é, conseguimos enxergar a derivada como uma transformação linear que aproxima a variação de uma função para dx pequeno. Esta é uma forma mais elegante de ver a derivada de uma função tanto para funções de uma, como de mais de uma variável. 
Diagrama 3- Curva de referência para o estabelecimento da derivada

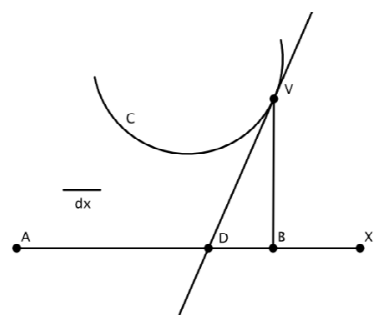

Adaptação livre (LEIBNIZ, 1995, p.105)

Em vista do que já foi discutido, nossa questão pode ser aqui recolocada de outro modo. Teria sido possível para Leibniz desenvolver o cálculo se nos elementos já não existisse algo que o permitisse fazê-lo? Recordando, nas proposições 7 e 8 do elemento 2, observamos que Euclides poderia ter dividido o quadrado em infinitas partes, mas não o fez porque esta não era a ideia reinante na época. Mesmo assim, Leibniz fez uso dos resultados de Euclides, pois as proposições ligadas ao traçado de tangentes parecem ser uma tônica em seus escritos. Destas muitas proposições de Euclides destacamos as proposições 17 e 18 do elemento 3, visto que nelas encontramos os embriões do método da tangente utilizado por Leibniz na construção e demonstração do funcionamento de seus cálculos.

\subsection{Proposição 17}

Dado um círculo U e um ponto A fora do círculo, construir uma reta que passe por $\mathrm{A}$ e tangencie o círculo U. (EUCLIDES, 2009, p.167)
Consideremos o círculo U com centro B e o ponto A fora do círculo como no Diagrama 4, então:

1. Construímos o segmento $A B$ que corta $\mathrm{U}$ em um ponto $\mathrm{C}$.

2. Traçamos uma perpendicular a $A B$ pelo ponto C. Esta reta corta o círculo $\mathrm{W}$ em um ponto $\mathrm{G}$.

3. Construímos o segmento BG cortando o círculo $\mathrm{U}$ em um ponto E.

Desse modo, a reta $\mathrm{AE}$ é a reta com o atributo desejado. Isto segue do seguinte argumento: como os segmentos $\mathrm{BC}$ e BE são iguais, assim como os segmentos BA e BG, então o triângulo AEB é congruente ao triângulo GCB, pois tem dois lados côngruos e o ângulo entre estes lados comum. Assim sendo, os ângulos AEB e GCB são iguais, mas GCB é reto, logo, o ângulo AEB é reto e segue-se então o resultado. $Q E D$.

Diagrama 4- Proposição 17 do livro 3 dos Elementos de Euclides

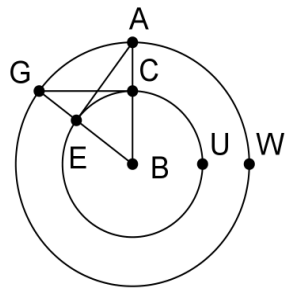

Adaptação livre (EUCLIDES, 2009, p.167)

Para traçar uma reta tangente a um círculo dado a partir de um ponto dado $\mathrm{A}$, foi feita a escolha de uma figura com duas circunferências concêntricas, sendo a reta 
traçada na circunferência menor e o ponto A localizado na circunferência maior. Com o centro no ponto $\mathrm{B}$, traçamos dois raios da circunferência maior BA e BG e fizemos a reta CG perpendicular à reta BA. Por congruência de triângulos, concluímos que AEB é igual a GCB e iguais ao ângulo reto. Como a reta $\mathrm{AE}$ toca em um ponto da circunferência e é perpendicular ao raio, logo ela é tangente à circunferência.

\subsection{Proposição 18}

Se uma reta tangencia o círculo $V$, então a reta que liga o centro do círculo a este ponto de tangência forma ângulos reto com a reta tangente. (EUCLIDES, 2009, p.168)

Consideramos o círculo $\mathrm{V}$, com centro $\mathrm{E}$, e uma reta $\mathrm{AB}$ que tangencia o círculo em um ponto $\mathrm{C}$, conforme o Diagrama 5.

1. Trace o segmento EC.

2. Agora suponha que EC não faça ângulos retos com $\mathrm{AB}$.

3. Trace então o segmento ED tal que este faça ângulos retos com $\mathrm{AB}$.

4. Este segmento cortará o círculo em um ponto $\mathrm{F}$.

Consideremos agora o triângulo CDE. Como o ângulo EDC é reto, o ângulo ECD é menor que um reto e, portanto, o lado ED é menor que o lado EC. Assim, chegamos à conclusão que EF também é menor que EC, o que é um absurdo, pois ambos são raios do círculo $C$. Segue então o resultado. $Q E D$.
Diagrama 5- Proposição 18 do livro 3 dos Elementos de Euclides

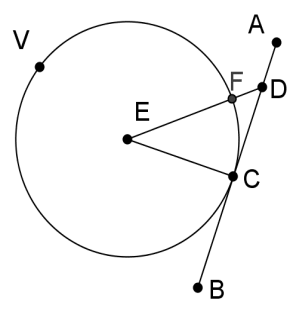

Adaptação livre (EUCLIDES, 2009, p.168)

A proposição 18 preocupa-se em mostrar que existindo uma reta tangente a um círculo, essa reta será perpendicular ao segmento que parte do centro (raio). Essa demonstração é um segmento da proposição 17 e é feita a partir da desigualdade triangular. Ela usa também a proposição que demonstra que ao maior ângulo de um triângulo opõe-se o maior lado.

A título de ilustração, algumas demonstrações de Euclides trazem para o leitor uma sensação de poderiam ser mais claras. A desigualdade triangular leva-nos a pensar que o ângulo OBC pode ser reto é verdadeira. No entanto, além de reto, este ângulo pode também ser obtuso ou agudo, desde que seja maior que os demais. Portanto, podemos mostrar o proposto através de ângulos na circunferência. Considerando o Diagrama 6, denomina-se $\mathrm{O}$, o centro da circunferência, $\log o \mathrm{AB}$ é o diâmetro que divide a circunferência em duas partes iguais. $\mathrm{O}$ ângulo $\mathrm{ABC}$ é o ângulo semi-inscrito $\mathrm{e}$ mede a metade do arco AKB, a medida de um reto. 
Diagrama 6- Clarificação da proposição 18 do livro 3 dos Elementos de Euclides

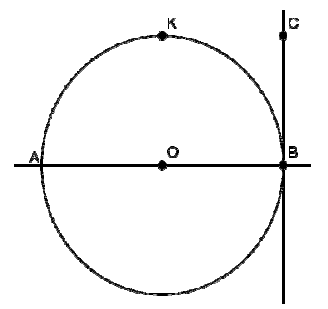

Adaptação livre (EUCLIDES, 2009, p.168)

É fato que todos os problemas de Leibniz envolvem processos infinitos e que Euclides não tinha clareza deste conhecimento, apesar de ter esbarrado nele algumas vezes. Euclides sabia que na natureza há processos que não podem ser completados com um número finito de passos e devem permanecer inacabados. Um bom exemplo disto seria a $\sqrt{2}$ pitagórica. Para Euclides, o segmento de reta que representa a $\sqrt{2}$ existia, mas sua representação numérica ainda era desconhecida; como também o fora para Pitágoras. (EVES, 2004, p.104-107). Igualmente infinitas eram as retas tangentes: nas proposições 17 e 18, se tomarmos dois pontos distintos em um círculo qualquer, podemos traçar uma reta secante passando por estes pontos. Se fizermos um destes pontos se mover ao longo da curva na direção do outro, a reta secante irá se mover na direção de uma reta tangente ao círculo neste ponto. Deste modo, o problema geométrico de encontrar uma reta tangente leva a um problema que envolve um processo infinito. Provavelmente, Euclides sabia disto. Ele só não sabia como implementar essa ideia.

\section{Conclusão}

A geometria euclidiana foi estabelecida para atender uma necessidade de entender e descrever a natureza. Com base nas imagens, na lógica e sem números, ela operou como uma facilitadora entre a natureza e a matemática. Nela, os métodos de análise e síntese são encontrados pela primeira vez e, por meio destes, a idéia de sintetizar imagens e analisá-las para provar proposições inaugurou uma escola que dura até os dias de hoje. Grosso modo, Euclides partiu do simples para o complexo e deste para o simples, fazendo um uso contínuo de resultados que ele mesmo havia demonstrado anteriormente. Suas imagens agem como suporte para que possamos chegar ao objetivo maior da Filosofia e da Matemática: alcançar a verdade. Euclides entendeu bem que nem todos compreendem o sentido das palavras, mas a maioria afina-se com a mensagem contida em uma imagem.

Retomaremos agora a questão que nos levou ao presente artigo, ou seja, mostrar que em algumas proposições dos treze elementos de Euclides encontram-se as primeiras notas do infinitamente pequeno de Leibniz e que sem este embrião, talvez não fosse possível para o filósofo alemão ter chegado tão longe. Primeiramente, concluímos que há indícios de que podemos pensar na questão do infinitamente pequeno em Euclides, uma vez que seu leitor é induzido a continuar dividindo os diagramas apresentados o quanto 
queira. Logo, a soma das partes divididas, resultaria na área original do diagrama. Portanto, há evidências que Euclides poderia ter pensado na questão do infinitamente pequeno. Porém, como os gregos não tinham esta noção clara, não podemos afirmar que Euclides pensou, de fato, em tal questão.

Mesmo assim, ficou patente que posteriormente Leibniz se inspirou nas proposições de Euclides, na subdivisão dos diagramas e na construção das retas tangentes para o estabelecimento dos cálculos infinitesimais.

\section{Referências}

EUCLIDES. Os Elementos. São Paulo: UNESP, 2009.

EVES, H. Introdução à História da Matemática. Campinas: UNICAMP, 2004.

HEIDEGGER, M. The principle of reason. Indianápolis: Indiana University Press, 1996.

\section{LEIBNIZ, G. W. Naissance du calcul}

différentiel: 26 articles des Acta eruditorum. Paris: Librarie Philosophique J. Vrin, 1995.

MOREAU, J. L'Univers Leibnizien. Paris: Emmanuel Vitte, 1956.

POMBO, O. Centro de Filosofia da Ciência da Universidade de Lisboa. Disponível em: (http://www.educ.fc.ul.pt/docentes/opombo/se minario/euclides/index.htm) Último Acesso: 23 de outubro de 2011.

1 Agradecemos ao Prof. Filadelfo Cardoso Santos ICT/UFVJM pelas pertinentes observações.

${ }^{2}$ Os debates acerca do infinito são anteriores a Platão e Aristóteles e foram uma constante nas escolas gregas. Foi durante o século V a.c. que Zenão de Eléa mostrou que se o conceito de contínuo e de infinita divisão for aplicado ao movimento de qualquer corpo, então o movimento não existe. Zenão expôs a sua argumentação com base em quatro situações hipotéticas, que ficaram conhecidas como os paradoxos de Zenão. Não existem registros na história que clarifiquem qual a razão que levou Zenão a desenvolver estes famosos argumentos. Talvez ele quisesse apenas ilustrar o pouco que sabemos sobre o tempo, o espaço, e tudo aquilo que não pode ser contado. De fato, depois da época de Zenão, a matemática não progrediu como se esperava. Nenhum dos problemas por ele propostos foi resolvido na antiguidade. Foi só durante o Renascimento, no séc. $\mathrm{XV}$, que o conceito de infinito reapareceu, tendo sido então amplamente estudado e discutido, embora muitas vezes faltasse alguma análise de detalhe e de raciocínio lógico. Giordano Bruno foi um dos mais importantes filósofos da renascença. $\mathrm{Na}$ sua obra Acerca do Infinito, do Universo e dos Mundos, o filósofo e religioso argumentou que o universo era infinito e que continha um número infinito de mundos, sistemas solares heliocêntricos, todos habitados por seres inteligentes. Seguindo seus passos, Galileu levantou a questão da equicardinalidade do conjunto dos inteiros e dos quadrados perfeitos. Nesta época, no entanto, os grandes debates e as grandes discussões centravam-se, não no infinito, mas sim nos infinitésimos que formaram a base de todo o cálculo infinitesimal. Por fim, coube ao matemático John Wallis, antecessor de Leibniz e Newton, a glória da criação do símbolo do infinito. (EVES, 2004, p.417-432)

3 Leibniz após estudar profundamente as obras de Cavalieri, Fermat, Pascal e Descartes chegou a seguinte conclusão, a saber: a determinação da tangente a uma curva dependia das diferenças das abscissas, $\mathrm{x}$, e ordenadas, y, na medida em que essas se tornassem infinitamente pequenas e que a quadratura, isto é a área, dependia da soma das ordenadas ou retângulos infinitamente finos. (MOREAU, 1956, p.118-120). Em junho de 1686, nosso filósofo publicou na Acta Erutitorum o artigo "De geometria recondita et analysi indivisibilium atque infinitorum" sobre o problema da quadratura como um caso especial do método do inverso das tangentes. (LEIBNIZ, 1995, p.126) 